
\title{
$\angle$ Research Square \\ The Risk of Bias in Iranian Randomized Controlled Trials Included in Cochrane Reviews
}

\section{Ali Kabir}

IUMS: Iran University of Medical Sciences

Ahmad Sofi-Mahmudi

Cochrane Iran

Arman Karimi Behnagh

Iran University of Medical Sciences

Vahid Eidkhani

Iran University of Medical Sciences

Hamid Reza Baradaran

Iran University of Medical Sciences

Payam Kabiri

Tehran University of Medical Sciences

Ali Akbar Haghdoost

Kerman University of Medical Sciences

Bita Mesgarpour ( $\nabla$ mesgarpour@research.ac.ir)

Cochrane Iran https://orcid.org/0000-0002-4004-8493

\section{Research article}

Keywords: Systematic review, evidence-based medicine, randomized controlled trial, Iran, Cochrane

Posted Date: September 22nd, 2020

DOI: https://doi.org/10.21203/rs.3.rs-76630/v1

License: (c) (1) This work is licensed under a Creative Commons Attribution 4.0 International License.

Read Full License 


\section{Abstract}

Background: Among interventional studies, randomized controlled trials (RCTs) provide the most conclusive evidence. However, RCTs can be susceptible to the risk of bias (RoB). Systematic reviews can be performed to appraise the RoB in the included articles using evaluative tools. This study aimed to describe the main characteristics and focus on the ROB of RCTs conducted in Iran and included in Cochrane Reviews (CRs).

Methods: We searched "Iran" by selecting the "Search All Text" and "Review" fields in the Cochrane Database of Systematic Reviews within Ovid. CRs that included the RCTs conducted in Iran were retrieved. A trial was selected only if it was included in CRs, described as a controlled clinical trial, involved human subjects and its RoB was assessed by CR authors. The trials were characterized by investigating the relevant articles and the table "Characteristics of included studies" in each CR. The RoB was investigated by collecting the judgments of the review authors made based on tables of RoB assessment in the CRs.

Results: Out of 1166 Iranian RCTs included by 571 CRs, a low RoB was found in $44.9 \%$ for random sequence generation, $20.8 \%$ for allocation concealment, $32.3 \%$ for blinding of participants/personnel, $36.5 \%$ for blinding of outcome assessors, $56.3 \%$ for incomplete outcome data, $41.3 \%$ for selective outcome reporting and $53.8 \%$ for other sources of bias.

Conclusion: The RoB in Iranian RCTs was found to be mostly high or unclear. It is therefore recommended that the methodological quality of RCTs be seriously addressed in Iran.

\section{Background}

Bias or systematic error can lead to over-reporting or under-reporting the treatment effects. Although randomised controlled trials (RCTs) are considered the gold standard for designing clinical research, their design, conduct, analysis and reporting are frequently at risk of flaws [1]. Moreover, results of systematic reviews, especially those focusing on interventional studies and including RCTs, mainly depend on the quality of RCTs [2]. Including low-quality RCTs in a systematic review can result in unreliable estimates of the effects [3]. Efforts made in the past decades to improve the quality of RCTs include developing the Consolidated Standards of Reporting Trials (CONSORT) [4]. Failing to observe these standards by journals or incomplete adherence to the guidelines after their adoption, providing inadequate trainings for researchers and poor practices and processes of research governance in place can influence the quality of conducting and reporting in trials [5-8].

The quality of RCTs has been frequently investigated by the journal $[9,10]$, the subject $[11,12]$, the publication year [13] and rarely by the country $[14,15]$. These studies have assessed the quality of reporting in trials mainly using the CONSORT statement and therefore relied on the information retrieved from the reports. 
Cochrane reviews (CRs) are considered the gold standard for systematic reviews owing to their implementation of the most stringent standards for quality assessment in terms of conduct and reporting [16]. CRs are publishing in the CDSR as the most distinguished journal and database of systematic reviews and meta-analyses in healthcare. As part of the Cochrane Library, the CDSR includes all CRs and protocols prepared by authors who register titles with a CR group [17]. Each CR group supports CR authors in methodological and editorial issues by focusing on a specific topic. The original Cochrane RoB tool recommended for the included RCTs in 2008 was updated in 2011 and revised in 2019 [2, 18].

The number of RCTs registered in the Iranian Registry of Clinical Trials (IRCT), which was established as a WHO primary registry at the end of 2008 [19] substantially rose to more than 25,400 in July 2020 . The poor quality of reporting in Iranian RCTs has been well investigated [20]. To the best of the authors' knowledge, this quality has not been evaluated yet in terms of the RoB. This evaluation can also help rank countries in terms of the quality of their RCTs.

This study was conducted to provide an overview of the characteristics and RoB in the RCTs conducted in Iran and included in CRs and to identify the areas requiring improvements the most.

\section{Methods}

We searched "Iran" by selecting the "Search All Text" and "Review" fields in the CDSR within Ovid on September 30, 2019. CRs were screened to identify those that included Iranian RCTs. The main characteristics extracted of the eligible CRs included DOI, publication year, CR Group, numbers of the included trials and Iranian trials and the total population of the included trials. A trial was selected only if it was included in CRs, described as a controlled clinical trial, involved human subjects and its RoB was assessed by CR authors.

Four independent authors, namely AK, AKB, VE and AS, extracted the characteristics of each trial by investigating the table "Characteristics of included studies" in each CR and the relevant published article in case the data provided were insufficient. The data collected associated with the features of the trials included the first author's name, the article's title, language and publication year, the name of the journal, the type and beginning and end dates, city and province of the intervention, the number of centers involved, the sample size, the allocation type and RoB assessment results. RCTs, quasi-experimental studies, crossover RCTs, cluster RCTs, non-randomized and others were considered RCT assignments. The allocation type was classified as treatment, supportive care, prevention, diagnostic and patient education. Treatment was defined any pharmaceutical intervention and supportive care was considered any other healthcare interventions from psychologists, rehabilitation, physiotherapy, occupational therapy, dietetics, complementary therapies as well as pain specialists and social workers [21].

CRs appraise the RoB in the included RCTs using a specific domain-based evaluative tool. The RoB was judged in each criterion as 'Low risk', 'High risk' or as 'Unclear risk', when there was a lack of information or uncertainty over the potential for bias. To describe RoB assessments, the judgments of the review authors were extracted for each criterion as an RoB assessment table in the CRs. The standard format of 
Cochrane RoB assessment comprises random sequence generation, allocation concealment, blinding of participants and personnels (performance bias), blinding of outcome assessors (detection bias), incomplete outcome data (attrition bias), selective outcome reporting and other sources of bias. An aggregate assessment was performed for the blinding reported in other formats such as subjective and objective outcomes or assessor, analyst, participants and/or caregivers. The blinding category was considered high-risk or low-risk bias if one or more subcategories of blinding items were reported as high or low risk bias. Otherwise, the blinding bias was reported as unclear. The diverse types of bias in each CR reported as funding bias, intention-to-treat bias, sample-size bias, for-profit bias and power calculation bias were aggregated as other sources of bias where appropriate. The RoB trend in Iranian RCTs was examined to explore the role of time in improving methodology and reporting. The present study did not assess the overall RoB given that this tool does not recommend evaluating this risk.

\section{Statistical analysis}

The extracted data were analysed in R version 3.6.0 (2019-04-26) (R Foundation for Statistical Computing, Vienna, Austria; http://www.R-project.org). The RCTs were characterized using frequency and relative frequency.

\section{Results}

The RoB of 1166 out of 1894 Iranian RCTs included in 571 retrieved CRs had been assessed. The RCTs in the retrieved CRs were excluded mainly due to being an ongoing type of trial $(20.6 \%)$ or their assessment being pending (20.6\%) (Fig. 1).

A total of $7.3 \%$ (2.7\% of the population) of 15,894 RCTs recruiting an entire population of $5,461,452$ and included in the CRs were conducted in Iran and their RoB had been assessed. Five CRs with an unknown population were, however, excluded from the analysis. A total of $63.7 \%$ of the CRs from 1970 to 2018 were produced by fifty CR groups after 2013 (Fig. 2). The majority of Iranian RCTs addressed pregnancy and childbirth $(n=250,21.4 \%)$, gynaecology and fertility $(n=158,13.5 \%)$ and skin and oral health $(n=70$, 6.0\%). A CR entitled "Interventions for old world cutaneous leishmaniosis" by members of the Cochrane Skin Group in Madrid published in 2017 included the highest number of Iranian RCTs, i.e. 27 out of a total of 49 (Heras-Mosteiro, 2017). No Iranian RCTs were reviewed by Cochrane HIV/AIDS, Lung Cancer, Methodology, STI and Urology groups. Two Iranian RCTs were included in two CRs and the RoB assessment performed in one of them was inconsistent.

The majority (81.0\%) of the study Iranian RCTs were performed between 2003 and 2016 in 60 cities. Figure 3 shows the heterogeneous distribution of location of the centers where the RCTs were performed, with the highest number performed in provinces of Tehran $(n=348,29.8 \%)$, Isfahan $(n=148,12.7 \%)$ and Fars $(n=97,8.3 \%)$. However, reporting was insufficient for the year of conducting study in 465 studies, the month of beginning of the trial in 283 further studies, the name of city in 107 studies and the number or name of the centers involved in the trial in 202 studies. 
These studies were published as article $(n=1149)$, abstract in conference proceedings $(n=10)$, IRCT registry $(n=6)$ and a PhD dissertation. The articles were published in in 521 peer-reviewed journals, mostly in English (90.1\%) and in domestic journals (33.6\%). Journal of Research in Medical Sciences, Isfahan University of Medical Sciences $(n=29)$, the International Journal of Gynecology \& Obstetrics $(n=$ 29) and the Iranian Red Crescent Medical Journal $(n=28)$ published the highest number of these articles.

The assignments were of an RCT type in 1040 (89.2\%) cases, a quasi-experimental type in $26(2.2 \%)$ and unclear in one study. The treatment (47.3\%) and supportive care $(24.2 \%)$ constituted the most common targets.

The sample size of the majority of the RCTs (83.6\%) was 30-200 with a median of 80, over 1000 in four of the studies and at most 50 in 25.5\%. The Cochrane Airways group reviewed an RCT with a sample size of 12514 as the maximum and the Public Health group an RCT with a sample size of 9 as the minimum. The full text of two of the articles was also inaccessible. Table 1 presents the sample size frequency.

Table 1

Frequency of sample size groups in Iranian RCTs

\begin{tabular}{|lll|}
\hline Sample size group & $\mathbf{N}(\%)$ & Cumulative \\
\hline $9-20$ & $34(2.9)$ & $34(2.9)$ \\
$21-40$ & $160(13.7)$ & $194(16.6)$ \\
$41-60$ & $232(19.9)$ & $426(36.5)$ \\
$61-80$ & $176(15.1)$ & $602(51.6)$ \\
$81-100$ & $169(14.5)$ & $771(66.1)$ \\
$101-150$ & $188(16.1)$ & $959(82.2)$ \\
$151-200$ & $87(7.4)$ & $1046(89.6)$ \\
\hline $201-300$ & $59(5.1)$ & $1105(94.7)$ \\
\hline $301-400$ & $29(2.5)$ & $1134(97.2)$ \\
\hline $401-500$ & $9(0.9)$ & $1149(98.1)$ \\
\hline $501-1000$ & $18(1.5)$ & $1161(99.6)$ \\
\hline $1001-12514$ & $4(0.4)$ & $1165(100)$ \\
\hline
\end{tabular}

From a methodological perspective, at least one arm was used as the control group in 1077 (92.4\%) studies, including 299 (25.6\%) that provided this group with a placebo. Moreover, 414 (35.5\%) studies were double-blinded, 28 (2.4\%) triple-blinded and 266 (22.8\%) used no blinding methods in their design. 
Not all the domains of the RoB tool were assessed in all the CRs; for instance, Cochrane reviewers had assessed random sequence generation in 1134 out of the 1166 RCTs and allocation concealment in 1122. Performance and attrition biases respectively received the highest frequency of high RoB (22.9\%) and low RoB (56.3\%). The RoB of at least one domain was judged as unclear in 931 (79.8\%) out of the 1166 included RCTs. Figure 4 shows the RoB assessed in the individual domains of the RoB tool for the included RCTs. According to Fig. 5, random sequence generation and incomplete outcome data continuously improved in terms of increasing low RoB during 2002-2017 (The analysis for all domains has been provided in the appendix 1).

\section{Discussion}

This study sought to investigate the quality of Iranian RCTs included in CRs based on the evaluations of Cochrane reviewers. To the best of the authors' knowledge, this study pioneered the quality evaluation of RCTs at a national scale using the Cochrane RoB tool. The quality of Iranian RCTs was found to be low in terms of the majority of RoB domains with a high or unclear RoB, which were mostly associated with the study design and included random sequence generation, allocation concealment and blinding.

A few studies mainly assessed RCTs of special subjects or fields using the CONSORT checklist and yielded consistent findings suggesting the poor methodological quality and reporting of the Iranian RCTs [22-25].

The present study found the RoB of $44.9 \%$ of the Iranian RCTs to be low in terms of random sequence generation, while the status of the remaining RCTs was impossible to be evaluated in terms of randomization given the inadequate data. Similarly, randomization was invalidated in $35.5 \%-98.7 \%$ of Iranian RCTs owing to their failure to report their randomization method, as was the case for the RCTs conducted in other countries [22, 26-28]; for instance, a low RoB was reported in terms of randomization for $44 \%$ of RCTs performed in Saudi Arabia [15]. A low RoB was also reported for below $50 \%$ of 1286 RCTs included in CRs.[29] Given that this problem is not specific to Iran, it is recommended that efforts be made at a global scale to enhance the quality of RCTs in this domain.

Blinding was assessed as two subdomains, i.e. blinding of participants and personnel (performance bias) and blinding of outcome assessors and analysts (detection bias). In terms of performance bias, only $32.3 \%$ ( $n=262$ ) of the included studies were assessed as low-risk, while 299 studies used placebos as the method of blinding their participants and personnel. This difference between the number of low-risk studies and that of studies using placebos shows the failure of Iranian researchers to comprehend the mechanism of implementing placebos to hide interventions from the participants or indicates their inability to properly explain the mechanism in a way that Cochrane reviewers are persuaded with the explanation. One-third of the studies were also evaluated as low-risk in terms of detection bias, which can be explained by the unwillingness of Iranians to participate in completely-blinded trials and failure to report the study details, which made it difficult for the reviewers to assess bias. Research generally suggests inadequately-performed blinding in the RCTs conducted in Iran and other countries [15, 29, 30]. 
A low RoB was assigned to attrition bias in $56.3 \%$ of cases, suggesting a low number of patients failing to follow up in the Iranian RCTs. In line with studies assessing Iranian RCTs in this domain, the present study found Iranian researchers to be relatively successful in providing data on the patients withdraw the study. Studies conducted in countries other than Iran have also reported the RoB of many RCTs as low in this domain $[15,29]$. A low RoB was assigned to selective outcome reporting in $436(41.3 \%)$ RCTs and an unclear RoB to 453 (42.9\%). This high frequency of unclear RoB can be explained by the failure of the majority of Iranian authors to register their studies in databases such as the IRCT, which resulted in failing to publish the study protocol before the final results were published. The reviewers had therefore difficulty evaluating this domain based on the outcomes reported in the article. Given the generallydifficult assessment of selective outcome reporting and other bias domains [31] these results should be cautiously interpreted.

The limitations of the Iranian RCTs included a small sample, which was below 60 in their majority. The distribution of the sites of conducting the RCTs was also heterogeneous and many of the studies were unicenter and conducted in Tehran, the capital of Iran, which can be explained by the significantly higher number of universities and top medical universities located in Tehran compared to in other cities and provinces. It is recommended that more collaborative, multi-center and high-quality studies be conducted by making proper research policies.

According to Chalmers and Glasziou, an estimated significant portion (85\%) of medical research is wasted in many dimensions and phases, namely relevance of the research question to the patients and physicians, appropriateness of the study design, accessibility of full text and unbiased and usable reporting. The present findings suggest the limitations of Iranian RCTs include all these four dimensions. Some of these issues, e.g. low-quality reporting, could be simply avoided. The introduction of the CONSORT statement in 1996 [4] has increased the percentage of studies with a low RoB in randomization, blinding of outcome assessors, incomplete data reporting and other sources of bias. However, the high frequency of unclear RoB in all the domains can be an alarming sign of low-quality reporting in Iranian RCTs.

The strengths of the present study include the evaluation of the RCTs by third-party reviewers with no prejudices or bias. The RoB was, however, differently reported in different CRs for the same RCT. This discrepancy was also raised in previous assessments of the RoB based on the Cochrane tool [32].

\section{Conclusions}

The present study revealed a long way ahead of Iranian researchers to conduct and report a welldesigned RCT. Despite the major improvements observed between initial and the most recent RCTs, a special attention should be paid to methodological trainings in certain domains such as allocation concealment and blinding.

\section{Abbreviations}


RCT: Randomized Controlled Trial; RoB: Risk of Bias; CR: Cochrane Reviews; CONSORT: Consolidated Standards of Reporting Trials; IRCT: Iranian Registry of Clinical Trials

\section{Declarations}

The Authors declare that there is no conflict of interest.

\section{Ethics approval and consent to participate}

The study has received ethical approval from the National Institute for Medical Research Development in Iran.

\section{Consent for publication}

Not applicable.

\section{Availability of data and materials}

All data and materials are available from the corresponding author upon request.

\section{Competing interests}

The authors declare they have no competing interests.

\section{Funding}

This work was partly supported by a research grant from National Institute for Medical Research Development in Iran (Grant Number: 962656).

\section{Authors' contributions}

$A K, H R B, P K, A H$ and $B M$ conceived the study and were responsible for the design and draft of the manuscript. ASM, AKB, VE and BM were responsible for data collection, analysis, interpretation and first draft of the manuscript. ASM conducted the analyses. All authors critically revised and approved the manuscript.

\section{Acknowledgements}

The authors would like to acknowledge and thank Maryam Hosseinzadeh for her help on retrieving included studies.

\section{References}

1. Higgins JP, Altman DG, Gøtzsche PC, Jüni P, Moher D, Oxman AD, Savović J, et al. The Cochrane Collaboration's tool for assessing risk of bias in randomised trials. BMJ. 2011; doi: 
10.1136/bmj.d5928.

2. Higgins JPT, Savović J, Page MJ, Elbers RG, Sterne JAC. Chapter 8: Assessing risk of bias in a randomized trial. In: Higgins JPT, Thomas J, Chandler J, Cumpston M, Li T, Page MJ, Welch VA (editors). Cochrane Handbook for Systematic Reviews of Interventions version 6.0 (updated July 2019). Cochrane, 2019. Available from www.training.cochrane.org/handbook.

3. Verhagen AP, De Vet HC, De Bie RA, Boers M, Van Den Brandt PA. The art of quality assessment of RCTs included in systematic reviews. J Clin Epidemiol. 2001;54(7):651-654.

4. Begg C, Cho M, Eastwood S, Horton R, Moher D, Olkin I, et al. Improving the quality of reporting of randomized controlled trials: the CONSORT statement. JAMA. 1996;276(8):637-639.

5. Gohel M, Chetter I. Are clinical trials units essential for a successful trial? BMJ. 2015; doi: 10.1136/bmj.h2823

6. Kane JM, Leucht S. Unanswered questions in schizophrenia clinical trials. Schizophr Bull. 2008;34(2):302-309.

7. Moher D, Schulz KF, Altman D, Consort Group. The CONSORT statement: revised recommendations for improving the quality of reports of parallel-group randomized trials. JAMA.2001;285(15):19871991.

8. Xu L, Li J, Zhang M, Ai C, Wang L. Chinese authors do need CONSORT: reporting quality assessment for five leading Chinese medical journals. Contemp Clin Trials Commun. 2008;29(5):727-731.

9. Lai TY, Wong VW, Lam RF, Cheng AC, Lam DS, Leung GM. Quality of reporting of key methodological items of randomized controlled trials in clinical ophthalmic journals. Ophthalmic Epidemiol. 2007;14(6):390-398.

10. Rios LP, Odueyungbo A, Moitri MO, Rahman MO, Thabane L. Quality of reporting of randomized controlled trials in general endocrinology literature. J Clin Endocr Metab. 2008; 93(10):3810-3816.

11. Lu L, Liao M, Zeng J, He J. Quality of reporting and its correlates among randomized controlled trials on acupuncture for cancer pain: application of the CONSORT 2010 Statement and STRICTA. Expert Rev Anticancer Ther. 2013;13(4):489-498.

12. Ziogas DC, Zintzaras E. Analysis of the quality of reporting of randomized controlled trials in acute and chronic myeloid leukemia, and myelodysplastic syndromes as governed by the CONSORT statement. Ann Epidemiol 2009;19(7):494-500.

13. Clark L, Schmidt U, Tharmanathan P, Adamson J, Hewitt C, Torgerson D. Poor reporting quality of key Randomization and Allocation Concealment details is still prevalent among published RCTs in 2011: a review. J Eval Clin Pract. 2013;19(4):703-707.

14. Nojomi M, Ramezani M, Ghafari-Anvar A. Quality of reports on randomized controlled trials published in Iranian journals: application of the new version of consolidated standards of reporting trials (CONSORT). Arch Iran Med 2013;16(1):20-22.

15. Rajab AM, Hamza A, Aldairi RK, Alaloush MM, Saquib J, Saquib N. Systematic review on the quality of randomized controlled trials from Saudi Arabia. Contemp Clin Trials Commun. 2019; doi:10.1016/j.conctc.2019.100441 
16. Sharif MO, Janjua-Sharif FN, Ali H, Ahmed F. Systematic reviews explained: AMSTAR-how to tell the good from the bad and the ugly. Oral Health Dent Manag. 2013;12(1):9-16.

17. Starr M, Chalmers I, Clarke M, Oxman AD. The origins, evolution, and future of The Cochrane Database of Systematic Reviews. Int J Tech Assess Health Care 2009;25(S1):182-195.

18. Sterne JAC, Savović J, Page MJ, Elbers RG, Blencowe NS, Boutron I, et al. RoB 2: a revised tool for assessing risk of bias in randomised trials. BMJ. 2019; doi:10.1136/bmj.14898.

19. Solaymani-Dodaran M, Khalili D, Hosseini H, Najafi L, Kamali K, et al. Iranian Registry of Clinical Trials two years on and the timing of registrations. J Evid Based Med. 2011;4(3):168-171.

20. Habib Agahi R, Navabi N, Shahravan A, Ghassemi A. Critical appraisal of reporting randomized clinical trials published in Iranian dental journals during 2003-2010. J Dent (Tehran). 2014;11(3):310318.

21. Ahmedzai SH, Walsh D. Palliative medicine and modern cancer care. Semin Oncol. 2000;27(1):1-6.

22. Adib-Hajbaghery M, Adib M, Eshraghi Arani N. Evaluating the quality of randomized trials published in Persian nursing journals with more than 10 years of publishing using the CASP checklist. Iran J Nursing. 2017;30(109):1-9.

23. Alamri HM, Alharbi F. Quality Assessment of Randomized Clinical Trials Reporting in Endodontic Journals: An Observational Study from 2012 to 2017. J Endod 2018; 44:1246-1250.

24. Faizi F, Tavallaee A, Rahimi A, Saburi A, Saghafinia M. Quality assessment of randomized control trials applied psychotherapy for chronic pains in iran: a systematic review of domestic trials. Iran Red Crescent Med J. 2014; doi: 10.5812/ircmj.15312.

25. Mehrazmay A, Karambakhsh A, Salesi M. Reporting Quality Assessment of Randomized Controlled Trials Published in Nephrology Urology Monthly Journal. Nephrourol Month. 2015; doi: 10.5812 /numonthly.28752.

26. Ayatollahi MT, Jafari $P$, Ghaem H. An evaluation of the quality of published clinical trials in Iranian medical journals during 2001-2004. J Babol Univ Medical Sci. 2005;7(4):64-70.

27. Hosseini S, Ahmadinia H, Rezaeian M. Evaluation of the Quality of Writing of the Title and Abstract of Randomized Controlled Clinical Trial Papers Published in the Journals of the Iran, s Universities of Medical Sciences in 2016, Based on the CONSORT Checklist: A Descriptive Study. J Rafsanjan Univ Med Sci. 2019;18(3):267-284.

28. Taghipour A, Shakeri MT, Yousefi R, Barzanouni S. Assessment of Randomized Controlled Clinical Trials articles in the Journal of Dental School, Mashhad University of Medical Sciences: Published 2003-2015. J Mashhad Dent. 2017;41(1):11-20.

29. Yordanov Y, Dechartres A, Porcher R, Boutron I, Altman DG, Ravaud P. Avoidable waste of research related to inadequate methods in clinical trials. BMJ. 2015; doi: 10.1136/bmj.h809.

30. Nagai K, Saito AM, Saito TI, Kaneko N. Reporting quality of randomized controlled trials in patients with HIV on antiretroviral therapy: a systematic review. Trials. 2017;18(1):625. 
31. Hartling L, Bond K, Vandermeer B, Seida J, Dryden DM, Rowe BH. Applying the risk of bias tool in a systematic review of combination long-acting beta-agonists and inhaled corticosteroids for persistent asthma. PloS One. 2011; doi:10.1371/journal.pone.0017242.

32. Hartling L, Hamm MP, Milne A, Vandermeer B, Santaguida PL, Ansari M, et al. Testing the risk of bias tool showed low reliability between individual reviewers and across consensus assessments of reviewer pairs. J Clin Epidemiol. 2013;66:973-981.

33. Jordan VM, Lensen SF, Farquhar CM. There were large discrepancies in risk of bias tool judgments when a randomized controlled trial appeared in more than one systematic review. J Clin Epidemiol. 2017;81:72-76.

\section{Figures}

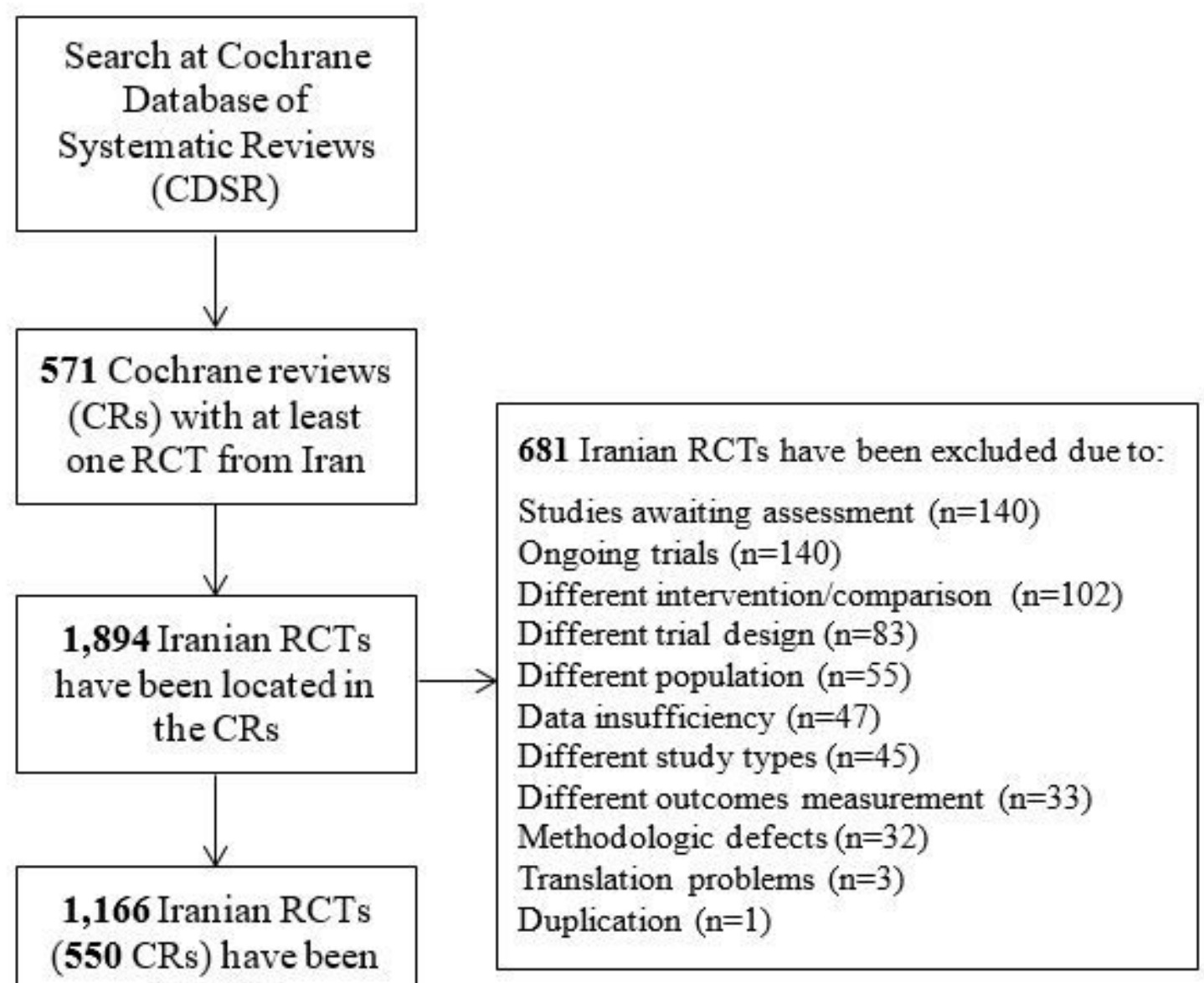

\section{Figure 1}

flow diagram of search for Iranian RCTs in Cochrane Reviews 


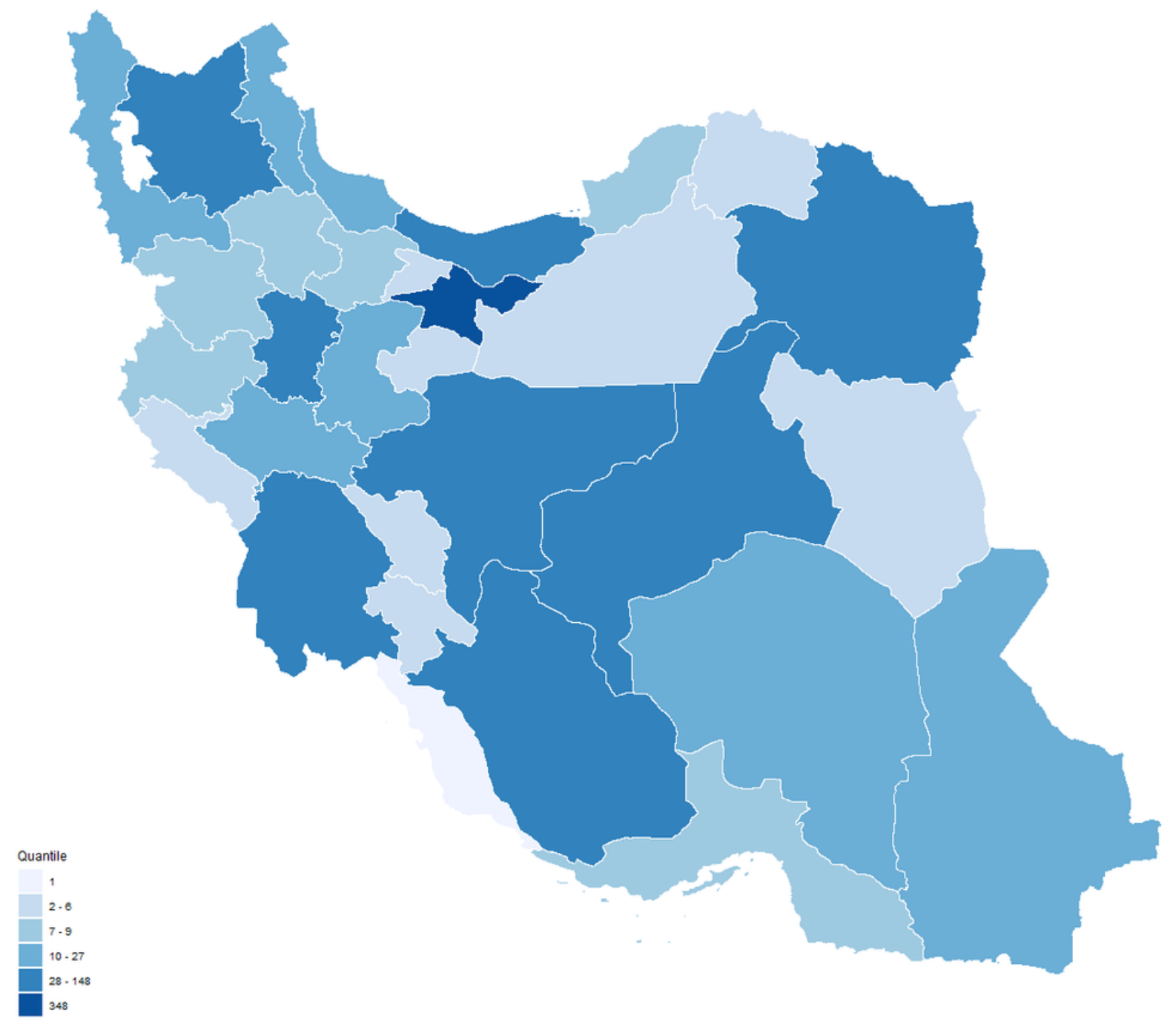

\section{Figure 2}

Distribution of conducting sites of Iranian RCTs The plot created using R package version 3.6.0 (2019-0426) (R Foundation for Statistical Computing, Vienna, Austria; http://www.R-project.org). Note: The designations employed and the presentation of the material on this map do not imply the expression of any opinion whatsoever on the part of Research Square concerning the legal status of any country, territory, city or area or of its authorities, or concerning the delimitation of its frontiers or boundaries. This map has been provided by the authors. 


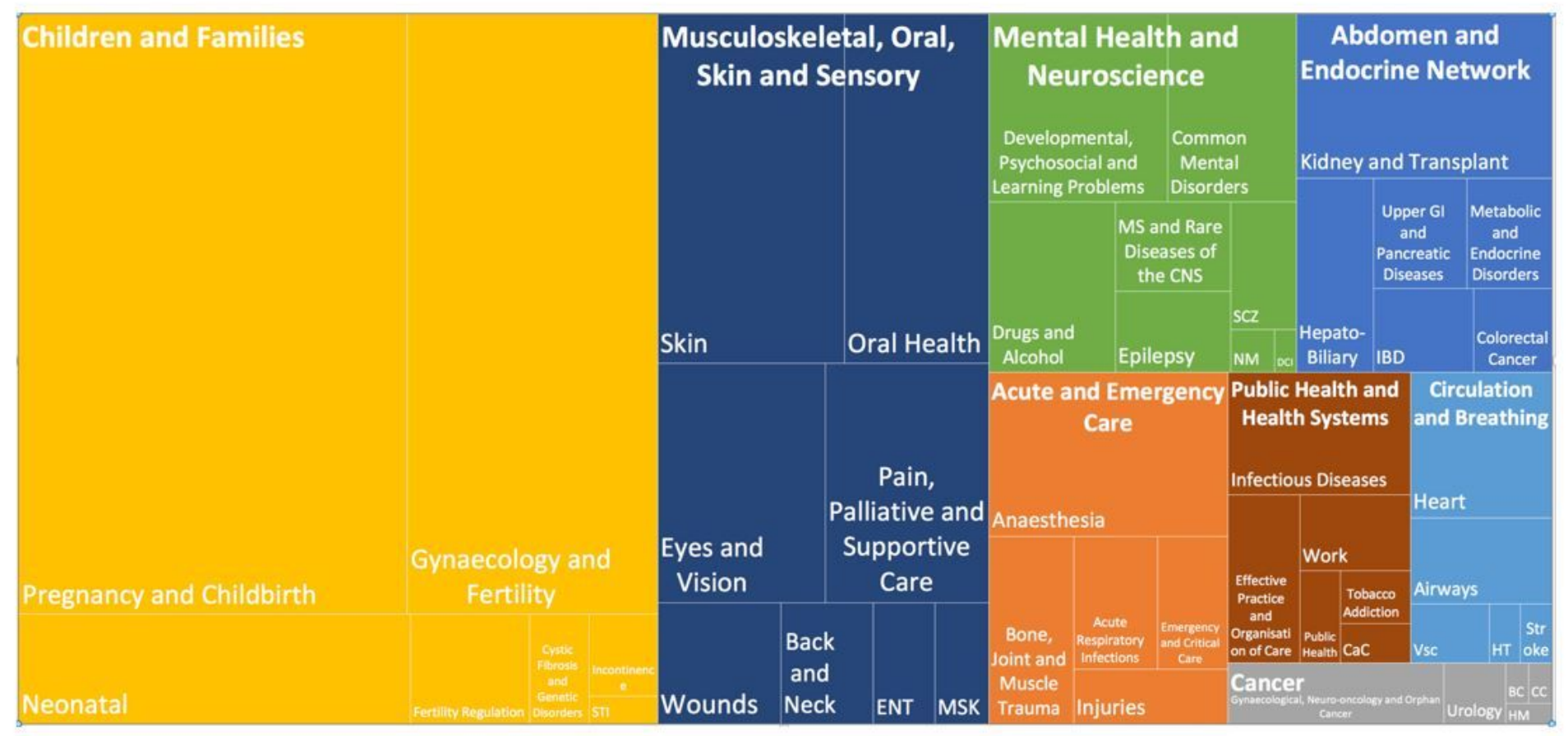

\section{Figure 3}

Distribution of Iranian RCTs in the Cochrane reviews based on Cochrane Review Groups BC: Breast Cancer; CaC: Consumers and Communication; CC: Childhood Cancer; DCl: Dementia and Cognitive Improvement; HM: Haematological Malignancies; HT: Hypertension; MSK: musculoskeletal; NM: Neuromuscular; SCZ: Schizophrenia; Vsc: Vascular 
Other bias

Selective reporting

Incomplete outcome data

Blinding (detection bias)

Blinding (performance bias)

Blinding (performance bias and detection bias)

Allocation concealment

Random sequence generation

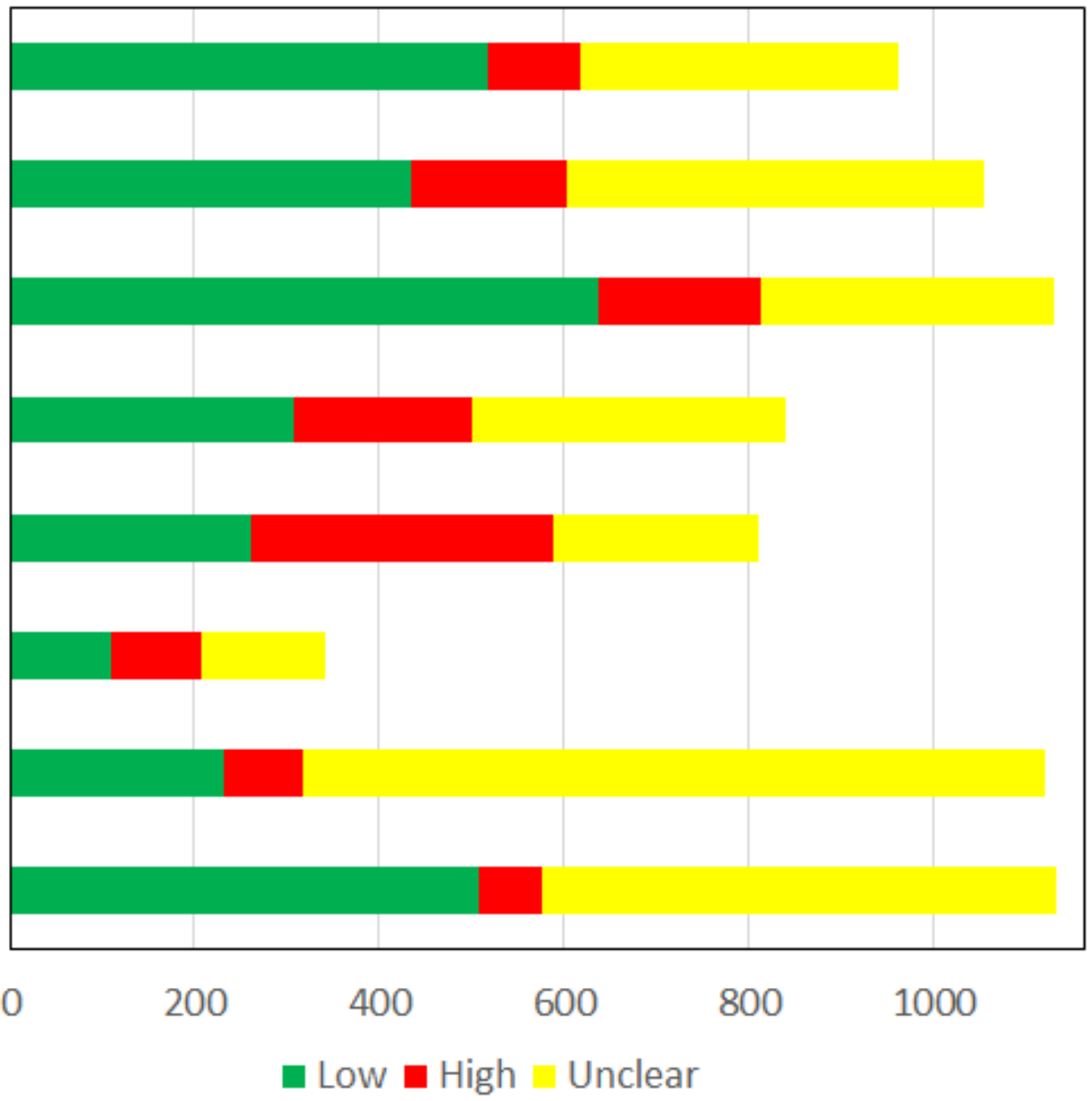

Figure 4

Overall Risk of Bias assessment of Iranian RCTs included and evaluated in Cochrane Reviews
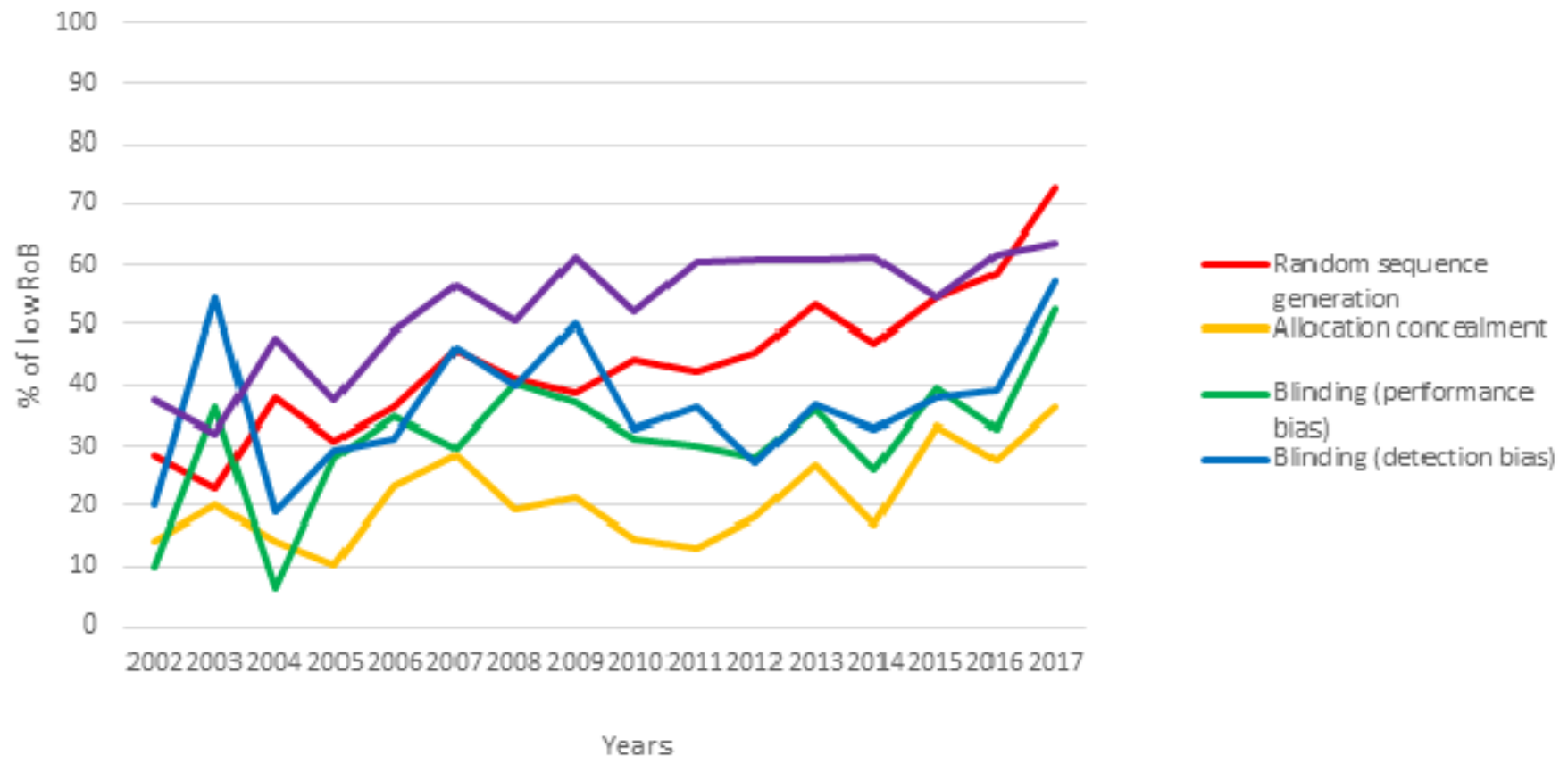
Figure 5

The trend of low RoB for four domains of Cochrane tool in the Iranian RCTs during 2002-2017

\section{Supplementary Files}

This is a list of supplementary files associated with this preprint. Click to download.

- 8.appendix1.docx 